\title{
True Neutrality as a New Type of Flavour
}

\author{
Rasulkhozha S. Sharafiddinov \\ Institute of Nuclear Physics, Uzbekistan Academy of Sciences, \\ Tashkent, 100214 Ulugbek, Uzbekistan
}

\begin{abstract}
A classification of leptonic currents with respect to C-operation requires the separation of elementary particles into the two classes of vector C-even and axial-vector C-odd character. Their nature has been created so that to each type of lepton corresponds a kind of neutrino. Such pairs are united in families of a different C-parity. Unlike the neutrino of a vector type, any C-noninvariant Dirac neutrino must have his Majorana neutrino. They constitute the purely neutrino families. We discuss the nature of a corresponding mechanism responsible for the availability in all types of axial-vector particles of a kind of flavour which distinguishes each of them from others by a true charge characterized by a quantum number conserved at the interactions between the C-odd fermion and the field of emission of the corresponding types of gauge bosons. This regularity expresses the unidenticality of truly neutral neutrino and antineutrino, confirming that an internal symmetry of a C-noninvariant particle is described by an axial-vector space. Thereby, a true flavour together with the earlier known lepton flavour predicts the existence of leptonic strings and their birth in single and double beta decays as a unity of flavour and gauge symmetry laws. Such a unified principle explains the availability of a flavour symmetrical mode of neutrino oscillations.
\end{abstract}

\section{Introduction}

There is no doubt that a notion about neutrino oscillation is based at first on the availability of solar neutrino problem but not on the absence of flavour symmetry. However, over fifty years ago, when the hypothesis of neutrino oscillation [1] was formulated for the first time and now, when a mixing of three types of neutrinos is observed [2,3], either the laboratory facts or the theoretical models are not in state to give a categorical answer to the question of whether or not an unbroken flavour symmetry exists. The answer is, as will be seen from the further, still written in matter fields [4].

According to old presentations about lepton nature, their interaction with the field of emission has been described as the vector $V_{l}$ current

$$
j_{e m}^{V_{l}}=\bar{u}\left(p^{\prime}, s^{\prime}\right)\left[\gamma_{\mu} f_{1 l}\left(q^{2}\right)-i \sigma_{\mu \lambda} q_{\lambda} f_{2 l}\left(q^{2}\right)\right] u(p, s),
$$

characterized by the CP-invariant components are the electric $f_{1 l}$ charge and its dipole $f_{2 l}$ moment [5]. After the opening a violation of parity [6] conservation concerning $\mathrm{P}$, the possibility of the availability has become clear between the lepton and the field of emission of one more another type of connection. This second type of interaction may be expressed in the form of an axial-vector $A_{l}$ current

$$
j_{\text {em }}^{A_{l}}=\bar{u}\left(p^{\prime}, s^{\prime}\right) \gamma_{5}\left[\gamma_{\mu} g_{1 l}\left(q^{2}\right)-i \sigma_{\mu \lambda} q_{\lambda} g_{2 l}\left(q^{2}\right)\right] u(p, s) .
$$

Here as well as in (11) $\sigma_{\mu \lambda}=\left[\gamma_{\mu}, \gamma_{\lambda}\right] / 2, q=p-p^{\prime}, p(s)$ and $p^{\prime}\left(s^{\prime}\right)$ denote the four-momenta (helicities) of incoming and outgoing particles. 
In quantum electrodynamics, it is usually accepted that the density of the photon leptonic current has the form

$$
j_{e m}=j_{e m}^{V_{l}}+j_{e m}^{A_{l}} .
$$

It includes the CP-invariant classical $g_{1 l}$ anapole $[7,8]$ and the CP-noninvariant electric $g_{2 l}$ dipole $[9,10]$ having a different T-symmetricality with the same P-antisymmetricality. Such a definition, however, leads to difficulties. In fact, it follows from considerations of symmetry that each C-even or C-odd dipole must arise as a result of a kind of charge [11].

In these circumstances, the processes on the nuclear targets may relate the mass $m_{l}$ to electric $f_{1 l}$ charge and dipole $f_{2 l}$ moment of any lepton as a unity of interaction structural parts. This connection in the low energy limit $\left(q^{2} \rightarrow 0\right)$ is reduced to one [12-15] of highly important consequences of the ideas of flavour symmetry:

$$
f_{1 l}(0)-2 m_{l} f_{2 l}(0)=0 .
$$

To express these ideas more clearly, one must refer to the case when to the process responds only the current axial-vector component. At such a situation, an equality of the interaction cross sections with the field of emission of both types of axial-vector $A_{l}$ currents may serve as a certain indication $[16,17]$ to an explicit connection

$$
g_{1 l}(0)-m_{l} g_{2 l}(0)=0 .
$$

In the presence of all parts of the interaction (3), the corresponding cross section for the process with polarized leptons includes not only self interference components $f_{i l}^{2}$ and $g_{i l}^{2}$, but also the contributions $\lambda_{c} s f_{1 l} g_{1 l}$ and $s f_{2 l} g_{2 l}$ of the mixed interference [18-20] between the two currents of the vector and axial-vector character. Among them $\lambda_{c} s f_{1 l} g_{1 l}$ for the particle $\left(\lambda_{c}=+1\right)$ and the antiparticle $\left(\lambda_{c}=-1\right)$ are different. A given circumstance expresses the C-antisymmetry of CP-even anapole. In contrast to this, the absence of $\lambda_{c}$ reflects, in the case of $f_{2 l} g_{2 l}$, the fact that $g_{2 l}$ was accepted in (2) as the usual C-invariant dipole.

Thus, the mixed interference parts of the interaction cross section would seem to say in favor between $f_{i l}(0)$ and $g_{i l}(0)$ of a flavour symmetrical connection. Such a dependence, however, does not exist at all. The point is that the availability of the multipliers $\lambda_{c}$ and $s$ violates parity conservation concerning $\mathrm{C}$ and $\mathrm{P}$ as well as the above-noted equality of the interaction cross section with the field of emission of leptonic current structural components. This in turn implies the violation of lepton number conservation law, confirming that we cannot exclude the validity of each of (4) and (5) only for particles of the defined type of lepton.

These facts give the right to interpret the classical $g_{1 l}$ anapole as the C-odd electric charge $[13,17]$. Therefore, if it turns out that $f_{i l}$ and $g_{i l}$ constitute symbolically the vector $V_{l}$ and axial-vector $A_{l}$ components of the same Dirac $(i=1)$ or Pauli $(i=2)$ part of leptonic current, from the point of view of (44) and (5), it should be expected that the dipole moments $f_{2 l}$ and $g_{2 l}$ correspond in nature to charges $[13,17]$ of vector $f_{1 l}$ and axial-vector $g_{1 l}$ types.

So, we have learned that any $\mathrm{C}$-invariant or $\mathrm{C}$-noninvariant charge may not serve as the source of the two types of the dipole moments of a different C-invariance. In other words, C-noninvariance of the anapole charge expresses the C-oddity as well as the CP-parity of an electric dipole. At the same time, T-noninvariance itself of an electric dipole is explained by the violation of T-parity conservation of the anapole charge even at the absence of CPT-symmetry of all types of $A_{l}$ currents.

Many authors state that a violation of CPT-symmetry is incompatible with the Lorentz invariance [21]. If we accept this idea, the absence of Lorentz symmetry would lead us to an extension [22] of the standard model [23-25]. 
Another characteristic moment is that regardless of whether [21] or not [26] a connection between the CPT and the Lorentz invariance exists, each neutrino [17,27] may not be simultaneously both a CP-even Dirac fermion and a CP-odd Majorana one [28]. At the same time, any type of charge cannot interact with all of the existing types of fields.

Therefore, the nature itself unites all elementary particles in families of a different Cinvariance. To one of them apply the C-invariant leptons $\left(l^{V} \neq \bar{l}^{V}\right)$ and their Dirac neutrinos $\left(\nu_{l}^{V} \neq \bar{\nu}_{l}^{V}\right)$ with vector $V_{l}$ currents. In these particles, the axial-vector interactions are absent. Among the fermions of another family one can meet the C-odd Majorana $\left(\nu_{M}^{A}=\bar{\nu}_{M}^{A}\right)$ neutrinos. They have their C-noninvariant Dirac $\left(\nu_{l}^{A}=\bar{\nu}_{l}^{A}\right)$ neutrinos [27] corresponding in united families to the kind of truly neutral leptons $\left(l^{A}=\bar{l}^{A}\right)$ of axial-vector $A_{l}$ currents. These particles do not possess the vector interactions. Such a separation of fermions into the two classes of vector $(V)$ and axial-vector $(A)$ types [13] leads us to a unified principle that

$$
\begin{gathered}
l^{V}=e^{V}, \mu^{V}, \tau^{V}, \ldots \rightarrow \nu_{l}^{V}=\nu_{e}^{V}, \nu_{\mu}^{V}, \nu_{\tau}^{V}, \ldots \\
l^{A}=e^{A}, \mu^{A}, \tau^{A}, \ldots \rightarrow \nu_{l}^{A}=\nu_{e}^{A}, \nu_{\mu}^{A}, \nu_{\tau}^{A}, \ldots \\
\nu_{D}^{A}=\nu_{e}^{A}, \nu_{\mu}^{A}, \nu_{\tau}^{A}, \ldots \rightarrow \nu_{M}^{A}=\nu_{1}^{A}, \nu_{2}^{A}, \nu_{3}^{A}, \ldots
\end{gathered}
$$

The feature of a presentation (66) is the idea that each type of vector lepton testifies in favor of the existence of a kind of C-invariant neutrino. These pairs can constitute the leptonic families of a vector nature. We must, therefore, define [29] their family structure in the form

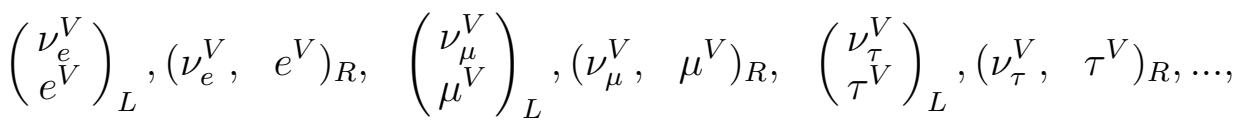

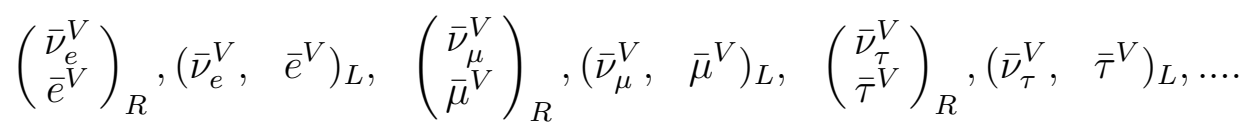

Any family possesses an individual flavour [30,31]. This gives the right to characterize each particle by the three $\left(l^{V}=e^{V}, \mu^{V}, \tau^{V}\right)$ lepton flavours:

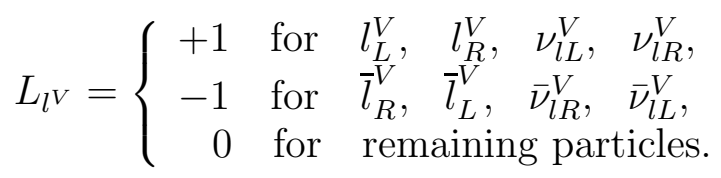

In the framework of a conservation law of full lepton number

$$
L_{e^{V}}+L_{\mu^{V}}+L_{\tau^{V}}=\text { const }
$$

or of all forms of lepton flavours

$$
L_{l^{V}}=\text { const, }
$$

a formation [29] of the left (right)-handed individual paraparticles

$$
\begin{array}{cc}
\left(l_{L}^{V}, \bar{l}_{R}^{V}\right), & \left(l_{R}^{V}, \bar{l}_{L}^{V}\right), \\
\left(\nu_{l L}^{V}, \bar{\nu}_{l R}^{V}\right), & \left(\nu_{l R}^{V}, \bar{\nu}_{l L}^{V}\right)
\end{array}
$$

at the interaction of C-invariant leptons or neutrinos with virtual photons of a vector nature $\left(\gamma^{V}\right)$ is fully possible. For example, in the elastic processes of the scattering on a nucleus Coulomb $(Z)$ charge

$$
l_{L, R}^{V}\left(\bar{l}_{R, L}^{V}\right)+A(Z) \stackrel{\gamma^{V}}{\rightarrow} l^{V}\left(\bar{l}^{V}\right)+A(Z),
$$




$$
\nu_{l L, R}^{V}\left(\bar{\nu}_{l R, L}^{V}\right)+A(Z) \stackrel{\gamma^{V}}{\rightarrow} \nu_{l}^{V}\left(\bar{\nu}_{l}^{V}\right)+A(Z) .
$$

Any difermion here can also explain the conservation of summed size of the C-even electric charge $e_{l^{V}}=f_{1 l^{V}}(0)$.

The presentation (7) expresses the regularity that each of axial-vector leptons has his truly neutral neutrino. They constitute the united families of C-noninvariant fermions of a Dirac nature. Thereby, such pairs establish their family structure:

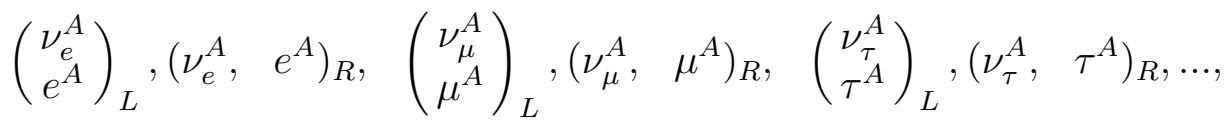

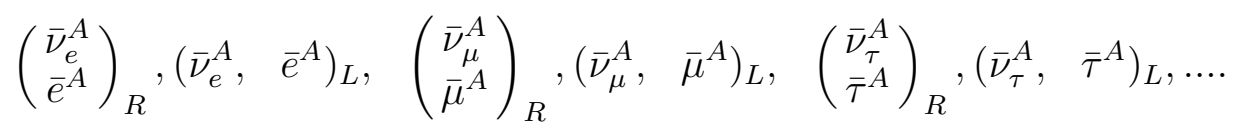

A highly characteristic feature of this picture is the fact that a formation of any of individual parafermions

$$
\begin{array}{cc}
\left(l_{L}^{A}, \bar{l}_{R}^{A}\right), & \left(l_{R}^{A}, \bar{l}_{L}^{A}\right), \\
\left(\nu_{l L}^{A}, \bar{\nu}_{l R}^{A}\right), & \left(\nu_{l R}^{A}, \bar{\nu}_{l L}^{A}\right)
\end{array}
$$

is responsible for conservation [17] of the summed size of the C-noninvariant electric charge $e_{l^{A}}=g_{1 l^{A}}(0)$, for example, in the processes on nuclear targets

$$
\begin{gathered}
l_{L, R}^{A}\left(\bar{l}_{R, L}^{A}\right)+A(Z) \stackrel{\gamma^{A}}{\rightarrow} l^{A}\left(\bar{l}^{A}\right)+A(Z), \\
\nu_{l L, R}^{A}\left(\bar{\nu}_{l R, L}^{A}\right)+A(Z) \stackrel{\gamma^{A}}{\rightarrow} \nu_{l}^{A}\left(\bar{\nu}_{l}^{A}\right)+A(Z),
\end{gathered}
$$

where the incoming fluxes consist of C-odd leptons or of their Dirac neutrinos. They interact with virtual axial-vector photons $\left(\gamma^{A}\right)$, the existence of which follows, as we will see below, from the local gauge invariance of the discussed types of fields.

Another important consequence implied from (8) is that to each type of C-noninvariant Dirac neutrino corresponds a kind of truly neutral Majorana neutrino. The similar pairs constitute the purely neutrino families of axial-vector fermions of a different nature. This gives the possibility to define their family structure [27] in the following form:

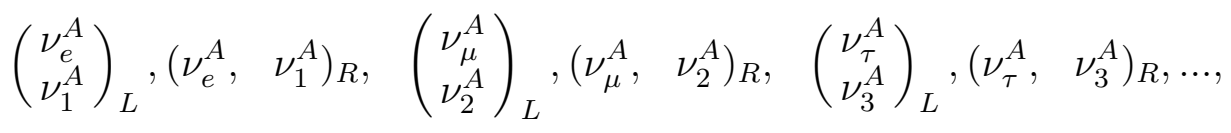

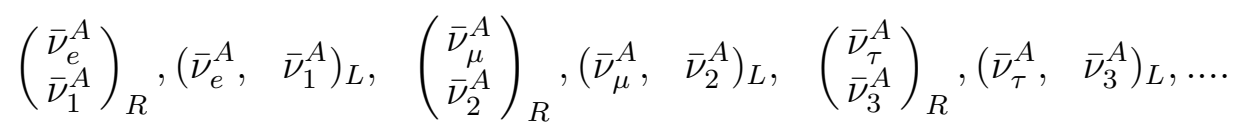

Such a picture is based logically on the idea of a coexistence law of truly neutral neutrinos of Dirac and Majorana types [27]. Thereby, it predicts the conservation of the C-noninvariant axial-vector electric charge [17], for example, in the elastic scattering on a spinless nucleus

$$
\nu_{M L, R}^{A}\left(\bar{\nu}_{M R, L}^{A}\right)+A(Z) \stackrel{\gamma^{A}}{\rightarrow} \nu_{M}^{A}\left(\bar{\nu}_{M}^{A}\right)+A(Z)
$$

as a unification of left (right)-handed C-odd fermions in individual difermions

$$
\left(\nu_{M L}^{A}, \bar{\nu}_{M R}^{A}\right), \quad\left(\nu_{M R}^{A}, \bar{\nu}_{M L}^{A}\right) .
$$

On the other hand, as was noted in the work [13], any of parafermions (14) and (15) can be formed only in the case where flavour symmetry establishes the interaction P-symmetrical 
picture, although P-symmetry of fermion is basically violated at the expense of its self inertial mass [27]. If we here take into account that as well as in the systems (14) and (15), the existence of a hard P-symmetrical connection between the two particles of each of paraparticles (20), (21) and (27) is by no means excluded naturally [17], there arises a question of whether the hypothesis about unidenticality of truly neutral neutrino and antineutrino is not strictly nonverisimilar even at the violation of CPT-symmetry of a CP-even axial-vector fermion itself.

Our purpose in a given work is to formulate a principle, according to which, any of families (18), (19), (24) and (25) distinguishes from others by a true charge characterized by a quantum number conserved in the processes with axial-vector leptons and neutrinos analogously to the fact that lepton flavours or full lepton number are conserved at the interactions between the vector fermion and the field of emission of the corresponding types of gauge bosons. The true charges of all $\mathrm{C}$-antisymmetrical leptons for both types of $\mathrm{C}$-noninvariant neutrinos and antineutrinos are different and that, consequently, lead to the kind of truly strict selection rules.

\section{From the earlier CP-symmetry to the new flavour}

A truly neutrality of neutrinos of a Majorana nature implies the absence in such fermions of a vector C-even electric charge and of each lepton flavour. But, as we have already mentioned above, unlike the earlier picture of the united Coulomb interactions with leptonic currents, their classification with respect to $\mathrm{C}$-operation assumed the existence for all $\mathrm{C}$-noninvariant leptons and neutrinos of a kind of axial-vector C-odd electric charge [17].

It is important that by this reason in the interaction process with nuclei of the two types of massive Dirac neutrinos of the vector $V$ and axial-vector $A$ nature

$$
\begin{gathered}
\nu\left(\nu_{l L, R}^{V}, \nu_{l L, R}^{A}\right)+A(Z) \stackrel{\gamma^{V}, \gamma^{A}}{\longrightarrow} \nu\left(\nu_{l}^{V}, \nu_{l}^{A}\right)+A(Z), \\
\bar{\nu}\left(\bar{\nu}_{l R, L}^{V}, \bar{\nu}_{l R, L}^{A}\right)+A(Z) \stackrel{\gamma^{V}, \gamma^{A}}{\longrightarrow} \bar{\nu}\left(\bar{\nu}_{l}^{V}, \bar{\nu}_{l}^{A}\right)+A(Z)
\end{gathered}
$$

there arise not only parafermions (15) and (21), but also the united systems of particles of a different C-invariance

$$
\begin{array}{ll}
\left(\nu_{l L}^{V}, \bar{\nu}_{l R}^{A}\right), & \left(\nu_{l R}^{V}, \bar{\nu}_{l L}^{A}\right), \\
\left(\nu_{l L}^{A}, \bar{\nu}_{l R}^{V}\right), & \left(\nu_{l R}^{A}, \bar{\nu}_{l L}^{V}\right) .
\end{array}
$$

The existence of any of paraneutrinos (30) and (31) is, as was mentioned in [13], incompatible with the parity concerning $\mathrm{C}$ and $\mathrm{P}$ as well as with the lepton number conservation law.

These violations would seem to say that either among the incoming particles the same Ceven or C-odd neutrinos are present or the hypothesis about unification of fermions in families of a different C-parity is not valid. On the other hand, as follows from simple reasoning, each type of an asymmetry [20] arising at the expense of interference between the interactions of the symmetrical and antisymmetrical character concerning $\mathrm{C}$ or $\mathrm{P}$, can be explained by a formation of a unified system of the two particles of the vector and axial-vector nature [32]. At the same time, nonconsevation of both $\mathrm{C}$ and $\mathrm{P}$ at the interaction of neutrinos of the most diverse currents with the field of emission is practically not excluded [33]. At our sight, this becomes possible owing to the fact that among the investigated and the used particles sometimes one can find as well as other types from the same elementary objects.

But here we have learned that an appearance in the nucleus charge field of any of paraparticles (30) and (31) explains once more the absence for all types of truly neutral leptons and neutrinos of each of the earlier known lepton flavours $\left(L_{l^{V}}\right)$ and the availability in them of a 
kind of flavour $\left(T_{l^{A}}\right)$ which can be called a true charge. Then it is possible, for example, the interaction with the field of emission of CP-invariant parafermions such as (30) and (31) is not forbidden by a conservation law

$$
L_{\nu_{l}^{V}}+T_{\bar{\nu}_{l}^{A}}=\text { const, } T_{\nu_{l}^{A}}+L_{\bar{\nu}_{l}^{V}}=\text { const. }
$$

According to these selection rules, any of difermions (30) and (31) may serve as the strong argument in favor of quantum numbers

$$
T_{\nu_{l}^{A}}=+1, \quad T_{\bar{\nu}_{l}^{A}}=-1 .
$$

Furthermore, each of paraneutrinos (15) and (27) there exists only in the presence of a unified P-symmetrical force between its structural particles. Such a connection appears as a consequence of a kind of regularity of nature of elastic scattering of vector Dirac and axial-vector Majorana neutrinos on nuclei

$$
\begin{aligned}
& \nu\left(\nu_{l L, R}^{V}, \nu_{M L, R}^{A}\right)+A(Z) \stackrel{\gamma^{V}, \gamma^{A}}{\longrightarrow} \nu\left(\nu_{l}^{V}, \nu_{M}^{A}\right)+A(Z), \\
& \bar{\nu}\left(\bar{\nu}_{l R, L}^{V}, \bar{\nu}_{M R, L}^{A}\right)+A(Z) \stackrel{\gamma^{V}, \gamma^{A}}{\longrightarrow} \bar{\nu}\left(\bar{\nu}_{l}^{V}, \bar{\nu}_{M}^{A}\right)+A(Z) .
\end{aligned}
$$

If this is so then from the point of view of any type of flavour $L_{l^{V}}$ or $T_{l^{A}}$, it should be expected that a formation of parafermions

$$
\begin{array}{ll}
\left(\nu_{l L}^{V}, \bar{\nu}_{M R}^{A}\right), & \left(\nu_{l R}^{V}, \bar{\nu}_{M L}^{A}\right), \\
\left(\nu_{M L}^{A}, \bar{\nu}_{l R}^{V}\right), & \left(\nu_{M R}^{A}, \bar{\nu}_{l L}^{V}\right)
\end{array}
$$

from the neutrinos of a different $\mathrm{C}$-invariance corresponds in united processes (34) and (35) to the existence of additive selection rules

$$
L_{\nu_{l}^{V}}+T_{\bar{\nu}_{M}^{A}}=\text { const, } T_{\nu_{M}^{A}}+L_{\bar{\nu}_{l}^{V}}=\text { const. }
$$

The latter together with ideas of each of paraparticles (36) and (37) predicts the size of a true charge for a Majorana neutrino

$$
T_{\nu_{M}^{A}}=+1, \quad T_{\bar{\nu}_{M}^{A}}=-1 .
$$

Therefore, It is relevant to emphasize once more the legality [17], for example, of interactions of the two types of axial-vector neutrinos of the Dirac and Majorana nature with the same field of emission

$$
\begin{aligned}
& \nu\left(\nu_{l L, R}^{A}, \nu_{M L, R}^{A}\right)+A(Z) \stackrel{\gamma^{A}}{\rightarrow} \nu\left(\nu_{l}^{A}, \nu_{M}^{A}\right)+A(Z), \\
& \bar{\nu}\left(\bar{\nu}_{l R, L}^{A}, \bar{\nu}_{M R, L}^{A}\right)+A(Z) \stackrel{\gamma^{A}}{\rightarrow} \bar{\nu}\left(\bar{\nu}_{l}^{A}, \bar{\nu}_{M}^{A}\right)+A(Z) .
\end{aligned}
$$

They can exist only in the case where the appearance of difermions (21) and (27) as well as of C-invariant paraneutrinos of the same P-parity

$$
\begin{array}{ll}
\left(\nu_{l L}^{A}, \bar{\nu}_{M R}^{A}\right), & \left(\nu_{l R}^{A}, \bar{\nu}_{M L}^{A}\right), \\
\left(\nu_{M L}^{A}, \bar{\nu}_{l R}^{A}\right), & \left(\nu_{M R}^{A}, \bar{\nu}_{l L}^{A}\right)
\end{array}
$$

from the C-odd fermions does not contradict the conditions

$$
T_{\nu_{l}^{A}}+T_{\bar{\nu}_{M}^{A}}=\text { const, } T_{\nu_{M}^{A}}+T_{\bar{\nu}_{l}^{A}}=\text { const. }
$$


Finally, insofar as a question about quantum numbers $T_{l^{A}}$ and $T_{\bar{l}^{A}}$, we will start from the elastic scattering of leptons $(l=e, \mu, \tau, \ldots)$ of a different $\mathrm{C}$-parity on the target nuclei

$$
\begin{aligned}
& l\left(l_{L, R}^{V}, l_{L, R}^{A}\right)+A(Z) \stackrel{\gamma^{V}, \gamma^{A}}{\longrightarrow} l\left(l^{V}, l^{A}\right)+A(Z), \\
& \bar{l}\left(\bar{l}_{R, L}^{V}, \bar{l}_{R, L}^{A}\right)+A(Z) \stackrel{\gamma^{V}, \gamma^{A}}{\longrightarrow} \bar{l}\left(\bar{l}^{V}, \bar{l}^{A}\right)+A(Z) .
\end{aligned}
$$

It originates only if unlike the individual dileptons (14) and (20), the united paraparticles

$$
\begin{array}{ll}
\left(l_{L}^{V}, \bar{l}_{R}^{A}\right), & \left(l_{R}^{V}, \bar{l}_{L}^{A}\right), \\
\left(l_{L}^{A}, \bar{l}_{R}^{V}\right), & \left(l_{R}^{A}, \bar{l}_{L}^{V}\right),
\end{array}
$$

appear jointly with the selection rules

$$
L_{l^{V}}+T_{\bar{l}^{A}}=\text { const, } \quad T_{l^{A}}+L_{\bar{l}^{V}}=\text { const. }
$$

Comparing their with that constitute difermions (14) and (20), but having in mind the paraleptons (47) and (48), one can found from (49) that

$$
T_{l^{A}}=+1, \quad T_{\bar{l}^{A}}=-1 .
$$

To verify the signs in sizes of (33), (39) and (50) one must apply to the processes with neutrinos of the most diverse types. A beautiful example is the muon decay of a vector nature

$$
\mu_{L, R}^{V} \stackrel{\gamma^{V}}{\rightarrow} e_{L, R}^{V} \bar{\nu}_{e R, L}^{V} \nu_{\mu L, R}^{V}, \quad \bar{\mu}_{R, L}^{V} \stackrel{\gamma^{V}}{\rightarrow} \bar{e}_{R, L}^{V} \nu_{e L, R}^{V} \bar{\nu}_{\mu R, L}^{V}
$$

These transitions say in favor of the C-even electric charge conservation

$$
\begin{aligned}
& e_{\mu^{V}}=e_{e^{V}}+e_{\bar{\nu}_{e}^{V}}+e_{\nu_{\mu}^{V}}, \\
& e_{\bar{\mu}^{V}}=e_{\bar{e}^{V}}+e_{\nu_{e}^{V}}+e_{\bar{\nu}_{\mu}^{V}},
\end{aligned}
$$

confirming that conditions

$$
\begin{gathered}
\Delta L_{\mu^{V}}=L_{\mu^{V}}-L_{\nu_{\mu}^{V}}=L_{e^{V}}+L_{\bar{\nu}_{e}^{V}}, \\
\Delta L_{\bar{\mu}^{V}}=L_{\bar{\mu}^{V}}-L_{\bar{\nu}_{\mu}^{V}}=L_{\bar{e}^{V}}+L_{\nu_{e}^{V}}
\end{gathered}
$$

correspond in (51) to a formation of vector difermions

$$
\begin{array}{ll}
\left(e_{L}^{V}, \bar{\nu}_{e R}^{V}\right), & \left(e_{R}^{V}, \bar{\nu}_{e L}^{V}\right), \\
\left(\bar{e}_{R}^{V}, \nu_{e L}^{V}\right), & \left(\bar{e}_{L}^{V}, \nu_{e R}^{V}\right) .
\end{array}
$$

To a similar implication one can lead starting from the $\mu^{A}$-decay

$$
\mu_{L, R}^{A} \stackrel{\gamma^{A}}{\rightarrow} e_{L, R}^{A} \bar{\nu}_{e R, L}^{A} \nu_{\mu L, R}^{A}, \quad \bar{\mu}_{R, L}^{A} \stackrel{\gamma^{A}}{\rightarrow} \bar{e}_{R, L}^{A} \nu_{e L, R}^{A} \bar{\nu}_{\mu R, L}^{A} .
$$

Here the appearance of axial-vector parafermions

$$
\begin{array}{cc}
\left(e_{L}^{A}, \bar{\nu}_{e R}^{A}\right), & \left(e_{R}^{A}, \bar{\nu}_{e L}^{A}\right), \\
\left(\bar{e}_{R}^{A}, \nu_{e L}^{A}\right), & \left(\bar{e}_{L}^{A}, \nu_{e R}^{A}\right)
\end{array}
$$


responds to conservation of both true number

$$
\begin{aligned}
\Delta T_{\mu^{A}} & =T_{\mu^{A}}-T_{\nu_{\mu}^{A}}=T_{e^{A}}+T_{\bar{\nu}_{e}^{A}}, \\
\Delta T_{\bar{\mu}^{A}} & =T_{\bar{\mu}^{A}}-T_{\bar{\nu}_{\mu}^{A}}=T_{\bar{e}^{A}}+T_{\nu_{e}^{A}},
\end{aligned}
$$

and C-odd electric charge

$$
\begin{aligned}
& e_{\mu^{A}}=e_{e^{A}}+e_{\bar{\nu}_{e}^{A}}+e_{\nu_{\mu}^{A}}, \\
& e_{\bar{\mu}^{A}}=e_{\bar{e}^{A}}+e_{\nu_{e}^{A}}+e_{\bar{\nu}_{\mu}^{A}} .
\end{aligned}
$$

Such a picture, however, loses the thought in decays

$$
\mu_{L, R}^{V} \stackrel{\gamma^{V}, \gamma^{A}}{\longrightarrow} e_{L, R}^{A} \bar{\nu}_{e R, L}^{A} \nu_{\mu L, R}^{V}, \quad \bar{\mu}_{R, L}^{V} \stackrel{\gamma^{V}, \gamma^{A}}{\longrightarrow} \bar{e}_{R, L}^{A} \nu_{e L, R}^{A} \bar{\nu}_{\mu R, L}^{V}
$$

where the paraparticles (59) and (60) appear owing to the selection rules

$$
\begin{aligned}
& \Delta L_{\mu^{V}}=L_{\mu^{V}}-L_{\nu_{\mu}^{V}}=T_{e^{A}}+T_{\bar{\nu}_{e}^{A}}, \\
& \Delta L_{\bar{\mu}^{V}}=L_{\bar{\mu}^{V}}-L_{\bar{\nu}_{\mu}^{V}}=T_{\bar{e}^{A}}+T_{\nu_{e}^{A}} .
\end{aligned}
$$

They satisfy the inequalities

$$
\begin{aligned}
& e_{\mu^{V}} \neq e_{e^{A}}+e_{\bar{\nu}_{e}^{A}}+e_{\nu_{\mu}^{V}}, \\
& e_{\bar{\mu}^{V}} \neq e_{\bar{e}^{A}}+e_{\nu_{e}^{A}}+e_{\bar{\nu}_{\mu}^{V}},
\end{aligned}
$$

which follow from the fact that a distinction of the truly neutral fermion and its antiparticle

$$
l^{A} \neq \bar{l}^{A}, \quad \nu_{l}^{A} \neq \bar{\nu}_{l}^{A}, \quad \nu_{M}^{A} \neq \bar{\nu}_{M}^{A}
$$

cannot take place with their respect to $\mathrm{C}$-operation, and is the consequence of an exact CPinvariance of a C-odd particle itself.

Thus, although dileptons (56) and (57) together with the conditions

$$
\begin{gathered}
e_{\mu^{A}} \neq e_{e^{V}}+e_{\bar{\nu}_{e}^{V}}+e_{\nu_{\mu}^{A}}, \\
e_{\bar{\mu}^{A}} \neq e_{\bar{e}^{V}}+e_{\nu_{e}^{V}}+e_{\bar{\nu}_{\mu}^{A}}
\end{gathered}
$$

indicate that

$$
\begin{aligned}
\Delta T_{\mu^{A}} & =T_{\mu^{A}}-T_{\nu_{\mu}^{A}}=L_{e^{V}}+L_{\bar{\nu}_{e}^{V}}, \\
\Delta T_{\bar{\mu}^{A}} & =T_{\bar{\mu}^{A}}-T_{\bar{\nu}_{\mu}^{T}}=L_{\bar{e}^{V}}+L_{\nu_{e}^{V}},
\end{aligned}
$$

their formation in the processes

$$
\mu_{L, R}^{A} \stackrel{\gamma^{V}, \gamma^{A}}{\longrightarrow} e_{L, R}^{V} \bar{\nu}_{e R, L}^{V} \nu_{\mu L, R}^{A}, \quad \bar{\mu}_{R, L}^{A} \stackrel{\gamma^{V}, \gamma^{A}}{\longrightarrow} \bar{e}_{R, L}^{V} \nu_{e L, R}^{V} \bar{\nu}_{\mu R, L}^{A}
$$

is forbidden by a charge conservation law.

Basing on the above-noted contradictions one can think that both $T_{l^{A}}$ and $T_{\bar{l}^{A}}$ in any of (33), (39) and (50) must have the opposite signs. In other words, charge nonconservation in the decays (65) and (75) assumed the existence of relations among the flavours

$$
\begin{gathered}
T_{l^{A}}=-L_{l^{V}}, \quad T_{\bar{l}^{A}}=-L_{\bar{l}^{V}}, \\
T_{\nu_{l}^{A}}=-L_{\nu_{l}^{V}}, \quad T_{\bar{\nu}_{l}^{A}}=-L_{\bar{\nu}_{l}^{V}} .
\end{gathered}
$$




$$
T_{\nu_{M}^{A}}=-L_{\nu_{l}^{V}}, \quad T_{\bar{\nu}_{M}^{A}}=-L_{\bar{\nu}_{l}^{V}} .
$$

From such a point of view, each of (66) and (67) can be leaded to his truly physical form

$$
\begin{array}{ll}
\Delta L_{\mu^{V}} \neq T_{e^{A}}+T_{\bar{\nu}_{e}^{A}}, \quad \Delta L_{\mu^{V}}=+ \text { const }, & T_{e^{A}}+T_{\bar{\nu}_{e}^{A}}=- \text { const }, \\
\Delta L_{\bar{\mu}^{V}} \neq T_{\bar{e}^{A}}+T_{\nu_{e}^{A}}, \quad \Delta L_{\bar{\mu}^{V}}=- \text { const }, & T_{\bar{e}^{A}}+T_{\nu_{e}^{A}}=+ \text { const },
\end{array}
$$

and equations (73) and (74) in this case are replaced by the inequalities

$$
\begin{aligned}
\Delta T_{\mu^{A}} \neq L_{e^{V}}+L_{\bar{\nu}_{e}^{V}}, \quad \Delta T_{\mu^{A}}=- \text { const }, & L_{e^{V}}+L_{\bar{\nu}_{e}^{V}}=+ \text { const }, \\
\Delta T_{\bar{\mu}^{A}} \neq L_{\bar{e}^{V}}+L_{\nu_{e}^{V}}, \quad \Delta T_{\bar{\mu}^{A}}=+ \text { const }, & L_{\bar{e}^{V}}+L_{\nu_{e}^{V}}=- \text { const } .
\end{aligned}
$$

Choices of types (76)-(178) establish in addition a flavour symmetrical picture of elastic scattering in which the absence of parity nonconservation connected with (32), (38) and (49) is explained by the availability of fundamentally important differences in the charges as well as in the masses [17] of vector and axial-vector fermions. Without loss of generality, we may write

$$
\begin{gathered}
e_{l^{A}} \neq e_{l^{V}}, \quad e_{\bar{l}^{A}} \neq e_{\bar{l}^{V}}, \\
e_{\nu_{l}^{A}} \neq e_{\nu_{l}^{V}}, \quad e_{\bar{\nu}_{l}^{A}} \neq e_{\bar{\nu}_{l}^{V}}, \\
e_{\nu_{M}^{A}} \neq e_{\nu_{l}^{V}}, \quad e_{\bar{\nu}_{M}^{A}} \neq e_{\bar{\nu}_{l}^{V}}, \\
m_{l^{A}} \neq m_{l^{V}}, \quad m_{\bar{l}^{A}} \neq m_{\bar{l}^{V}}, \\
m_{\nu_{l}^{A}} \neq m_{\nu_{l}^{V}}, \quad m_{\bar{\nu}_{l}^{A}} \neq m_{\bar{\nu}_{l}^{V}}, \\
m_{\nu_{M}^{A}} \neq m_{\nu_{l}^{V}}, \quad m_{\bar{\nu}_{M}^{A}} \neq m_{\bar{\nu}_{l}^{V}} .
\end{gathered}
$$

These consequences of flavour symmetry just and give the possibility to understand the hypothesis about unidenticality of fermions of the vector and axial-vector nature

$$
\begin{array}{cc}
l^{A} \neq l^{V}, & \bar{l}^{A} \neq \bar{l}^{V}, \\
\nu_{l}^{A} \neq \nu_{l}^{V}, & \bar{\nu}_{l}^{A} \neq \bar{\nu}_{l}^{V}, \\
\nu_{M}^{A} \neq \nu_{l}^{V}, & \bar{\nu}_{M}^{A} \neq \bar{\nu}_{l}^{V} .
\end{array}
$$

So, it is seen that a true number distinguishing a C-odd particle from his antiparticle is not of those lepton numbers, an introduction of which can be based on the unidenticality of neutrino and antineutrino of vector currents from the point of view of C-invariance of such fermions. At the same time, to any of both types of quantum numbers corresponds a kind of family. This reflects the availability of a unified regularity of their family structure depending on flavour dynamics of particles.

Thus, our presentations about the nature of truly neutral neutrinos allow to formulate the laws of a corresponding mechanism responsible for separation of axial-vector fermions among the other electroweakly and strongly interacted particles. They state that each of families (18), (19), (24) and (25) possesses his true flavour. We can, therefore, characterize any particle by the three $\left(l^{A}=e^{A}, \mu^{A}, \tau^{A}\right)$ true flavours:

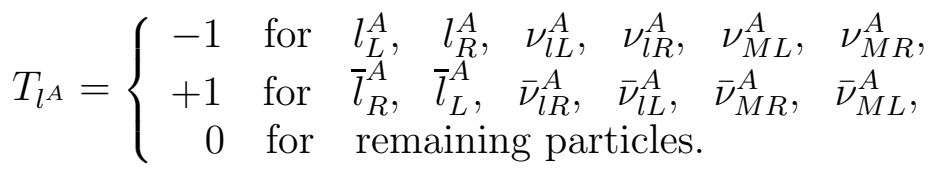


Conservation of full true number

$$
T_{e^{A}}+T_{\mu^{A}}+T_{\tau^{A}}=\text { const }
$$

or all types of true flavours

$$
T_{l^{A}}=\text { const }
$$

indicates to a principle that between the two left (right)-handed fermions from the families (18), (19), (24) and (25) there exists a unified force. It has an important consequence for the above-noted difermions of $A_{l}$ currents.

Of course, their appearance in the field of emission is compatible with the ideas of each of $\mathrm{C}$ and $\mathrm{P}$ as well as with the equality of summed charge of the interacted particles before and after the exchange by a virtual gauge boson. Such a principle is the unified and does not depend of whether the parafermions have a vector or an axial-vector nature. Then it is possible, for example, to interpret the P-symmetry as a conservation law not only of flavour $[12,13]$, but also of any type of the electric charge.

\section{Flavour symmetry criterion for lepton universality}

There exists a range of the structural phenomena in which the dynamical properties of Pparity become fully crucial. An example of this may be a decay of $\tau$-lepton of a vector nature

$$
\tau_{L, R}^{V} \stackrel{\gamma^{V}}{\rightarrow} e_{L, R}^{V} \bar{\nu}_{e R, L}^{V} \nu_{\tau L, R}^{V}, \quad \bar{\tau}_{R, L}^{V} \stackrel{\gamma^{V}}{\rightarrow} \bar{e}_{R, L}^{V} \nu_{e L, R}^{V} \bar{\nu}_{\tau R, L}^{V}
$$

As well as in (51), each of difermions (56) and (57) arises here at the conservation simultaneously of both lepton number

$$
\begin{aligned}
& \Delta L_{\tau^{V}}=L_{\tau^{V}}-L_{\nu_{\tau}^{V}}=L_{e^{V}}+L_{\bar{\nu}_{e}^{V}}, \\
& \Delta L_{\bar{\tau}^{V}}=L_{\bar{\tau}^{V}}-L_{\bar{\nu}_{\tau}^{V}}=L_{\bar{e}^{V}}+L_{\nu_{e}^{V}},
\end{aligned}
$$

and vector C-even electric charge

$$
\begin{aligned}
& e_{\tau^{V}}=e_{e^{V}}+e_{\bar{\nu}_{e}^{V}}+e_{\nu_{\tau}^{V}}, \\
& e_{\bar{\tau}^{V}}=e_{\bar{e}^{V}}+e_{\nu_{e}^{V}}+e_{\bar{\nu}_{\tau}^{V}} .
\end{aligned}
$$

The solutions (96)-(99) coincide with the corresponding sizes from (152)-(155) and that, consequently, the connections

$$
\begin{gathered}
L_{e^{V}}=L_{\mu^{V}}=L_{\tau^{V}}, \quad L_{\bar{e}^{V}}=L_{\bar{\mu}^{V}}=L_{\bar{\tau}^{V}}, \\
L_{\nu_{e}^{V}}=L_{\nu_{\mu}^{V}}=L_{\nu_{\tau}^{V}}, \quad L_{\bar{\nu}_{e}^{V}}=L_{\bar{\nu}_{\mu}^{V}}=L_{\bar{\nu}_{\tau}^{V}}
\end{gathered}
$$

say about lepton universality of a vector interaction

$$
\begin{aligned}
& e_{e^{V}}=e_{\mu^{V}}=e_{\tau^{V}}, \quad e_{\bar{e}^{V}}=e_{\bar{\mu}^{V}}=e_{\bar{\tau}^{V}}, \\
& e_{\nu_{e}^{V}}=e_{\nu_{\mu}^{V}}=e_{\nu_{\tau}^{V}}, \quad e_{\bar{\nu}_{e}^{V}}=e_{\bar{\nu}_{\mu}^{V}}=e_{\bar{\nu}_{\tau}^{V}} .
\end{aligned}
$$

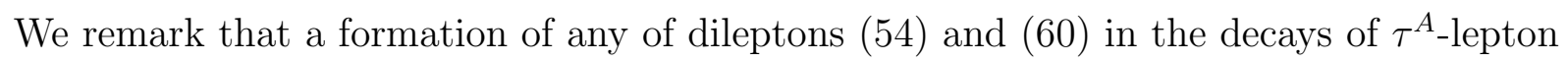

$$
\tau_{L, R}^{A} \stackrel{\gamma^{A}}{\rightarrow} e_{L, R}^{A} \bar{\nu}_{e R, L}^{A} \nu_{\tau L, R}^{A}, \quad \bar{\tau}_{R, L}^{A} \stackrel{\gamma^{A}}{\rightarrow} \bar{e}_{R, L}^{A} \nu_{e L, R}^{A} \bar{\nu}_{\tau R, L}^{A}
$$


is not forbidden by a selection rule

$$
\begin{aligned}
\Delta T_{\tau^{A}} & =T_{\tau^{A}}-T_{\nu_{\tau}^{A}}=T_{e^{A}}+T_{\bar{\nu}_{e}^{A}}, \\
\Delta T_{\bar{\tau}^{A}} & =T_{\bar{\tau}^{A}}-T_{\bar{\nu}_{\tau}^{A}}=T_{\bar{e}^{A}}+T_{\nu_{e}^{A}}
\end{aligned}
$$

and by a conservation law of an axial-vector $\mathrm{C}$-odd electric charge

$$
\begin{aligned}
& e_{\tau^{A}}=e_{e^{A}}+e_{\bar{\nu}_{e}^{A}}+e_{\nu_{\tau}^{A}}, \\
& e_{\bar{\tau}^{A}}=e_{\bar{e}^{A}}+e_{\nu_{e}^{A}}+e_{\bar{\nu}_{\tau}^{A}} .
\end{aligned}
$$

Their comparison with (61)-(64) leads us once more to an implication that

$$
\begin{aligned}
& T_{e^{A}}=T_{\mu^{A}}=T_{\tau^{A}}, \quad T_{\bar{e}^{A}}=T_{\bar{\mu}^{A}}=T_{\bar{\tau}^{A}}, \\
& T_{\nu_{e}^{A}}=T_{\nu_{\mu}^{A}}=T_{\nu_{\tau}^{A}}, \quad T_{\bar{\nu}_{e}^{A}}=T_{\bar{\nu}_{\mu}^{A}}=T_{\bar{\nu}_{\tau}^{A}},
\end{aligned}
$$

the existence of which can also be based simply on the lepton universality of an axial-vector interaction

$$
\begin{aligned}
& e_{e^{A}}=e_{\mu^{A}}=e_{\tau^{A}}, \quad e_{\bar{e}^{A}}=e_{\bar{\mu}^{A}}=e_{\bar{\tau}^{A}}, \\
& e_{\nu_{e}^{A}}=e_{\nu_{\mu}^{A}}=e_{\nu_{\tau}^{A}}, \quad e_{\bar{\nu}_{e}^{A}}=e_{\bar{\nu}_{\mu}^{A}}=e_{\bar{\nu}_{\tau}^{A}} .
\end{aligned}
$$

So, we can conclude that each type of flavour symmetry expresses the lepton universality of a kind of interaction as the one of dynamical aspects of its P-invariance.

\section{Family structure of nucleons}

Any of currents responsible for interactions of leptons and hadrons with virtual gauge bosons can symbolically be expressed in the form of a sum of vector and axial-vector parts of the same charge or dipole moment. This does not imply of course that the same neutron or proton must be simultaneously both a C-even and a C-odd nucleon. We have, thus, a full analogy to the fact that a classification of elementary particles with respect to $\mathrm{C}$-operation admits the existence of the two types of Dirac fermions of the vector $(V)$ and axial-vector $(A)$ nature. If such objects are the neutrons and protons, they will constitute the nucleonic $(N=n, p)$ families of doublets of a different C-invariance.

One group consisting of C-even nucleons of vector $V_{N}$ currents may be presented as

$$
\begin{aligned}
& \left(\begin{array}{l}
n^{V} \\
p^{V}
\end{array}\right)_{L},\left(n^{V}, p^{V}\right)_{R}, \\
& \left(\begin{array}{l}
\bar{n}^{V} \\
\bar{p}^{V}
\end{array}\right)_{R},\left(\bar{n}^{V}, \quad \bar{p}^{V}\right)_{L} .
\end{aligned}
$$

The second class includes the truly neutral C-odd nucleons of axial-vector $A_{N}$ currents

$$
\begin{aligned}
& \left(\begin{array}{l}
n^{A} \\
p^{A}
\end{array}\right)_{L},\left(n^{A}, \quad p^{A}\right)_{R}, \\
& \left(\begin{array}{l}
\bar{n}^{A} \\
\bar{p}^{A}
\end{array}\right)_{R},\left(\bar{n}^{A}, \quad \bar{p}^{A}\right)_{L} .
\end{aligned}
$$

For elucidation of their ideas, it is desirable to apply at first to the processes

$$
n_{L, R}^{V} \stackrel{\gamma^{V}}{\rightarrow} p_{L, R}^{V} \bar{e}_{R, L}^{V} \nu_{e L, R}^{V}, \quad \bar{n}_{R, L}^{V} \stackrel{\gamma^{V}}{\rightarrow} \bar{p}_{R, L}^{V} e_{L, R}^{V} \bar{\nu}_{e R, L}^{V}
$$


from which it follows that vector neutron and neutrino possess the same CP-odd electric charge

$$
e_{n^{V}}=e_{\nu_{e}^{V}}, \quad e_{\bar{n}^{V}}=e_{\bar{\nu}_{e}^{V}} .
$$

Exactly the same one can reanalyze the decays

$$
n_{L, R}^{A} \stackrel{\gamma^{A}}{\rightarrow} p_{L, R}^{A} \bar{e}_{R, L}^{A} \nu_{e L, R}^{A}, \quad \bar{n}_{R, L}^{A} \stackrel{\gamma^{A}}{\rightarrow} \bar{p}_{R, L}^{A} e_{L, R}^{A} \bar{\nu}_{e R, L}^{A}
$$

in conformity with the baryon [34] and true number conservation laws. In this case, it is expected that

$$
e_{n^{A}}=e_{\nu_{e}^{A}}, \quad e_{\bar{n}^{A}}=e_{\bar{\nu}_{e}^{A}},
$$

and thus, truly neutral neutron and neutrino do not distinguish from one another by the availability in them of an equal CP-even electric charge.

\section{From the flavour symmetry to the leptonic string}

The birth of a dilepton originates in any process of $\beta$-decay by the same reason. Such a reason can, for example, be existence in all leptonic families of a unified flavour symmetrical force between the two left (right)-handed fermions of each type. It establishes those connections, at which there exist the left (right)-handed leptons in difermions comparatively for a long time without converting into the right (left)-handed ones, although this is not forbidden. In other words, a flavour symmetrical force relates the two left (right)-handed leptons in flavourless dileptons. They are conserved in the form of leptonic strings until an external action is able to separate their by a part in the particle type dependence. Therefore, to understand the earlier known experimental facts at the fundamental dynamical level, one must use a principle that a single or a double $\beta$-decay is carried out in nuclei without neutrinos as well as without electrons by the schemes

$$
\begin{aligned}
n_{L, R} & \rightarrow p_{L, R}+\left(\bar{e}_{R, L}, \nu_{e L, R}\right), \\
\bar{n}_{R, L} & \rightarrow \bar{p}_{R, L}+\left(e_{L, R}, \bar{\nu}_{e R, L}\right), \\
2 n_{L, R} & \rightarrow 2 p_{L, R}+2\left(\bar{e}_{R, L}, \nu_{e L, R}\right), \\
2 \bar{n}_{R, L} & \rightarrow 2 \bar{p}_{R, L}+2\left(e_{L, R}, \bar{\nu}_{e R, L}\right) .
\end{aligned}
$$

In direct experiments left (right)-handed dileptons are observed instead of electrons. In addition, it is necessary to take into account the transitions

$$
\begin{aligned}
& \left(e_{L, R}, \bar{\nu}_{e R, L}\right) \leftrightarrow\left(e_{R, L}, \bar{\nu}_{e L, R}\right), \\
& \left(\bar{e}_{R, L}, \nu_{e L, R}\right) \leftrightarrow\left(\bar{e}_{L, R}, \nu_{e R, L}\right),
\end{aligned}
$$

because they correspond to the interconversions of nucleons of the different components.

Thus, all properties of electrons fixed in single or double $\beta$-decay one must consider as the characteristic features that refer doubtless only to a leptonic string that unites the electron and its antineutrino. Of course, any of them can also be observed in the form of a free particle. This, however, requires the creation devices of a sufficiently high sensitivity.

\section{Coulomb fields of a different nature}

One of the most highlighted features of a classification of elementary particles with respect to C-operation is its notion about axial-vector photons $\left(\gamma^{A}\right)$ having an electric nature. Their 
existence, as was mentioned earlier, may also be accepted as a consequence of invariance of the Dirac Lagrangian of a C-noninvariant lepton

$$
L_{0}^{A}=\bar{\psi}_{l^{A}} \gamma_{5}\left(i \gamma_{\mu} \partial_{\mu}-m_{l^{A}}\right) \psi_{l^{A}}
$$

concerning the local axial-vector gauge transformation

$$
\psi_{l^{A}}^{\prime}(x)=U_{A} \psi_{l^{A}}(x), \quad U_{A}=e^{i \beta(x) \gamma_{5}} .
$$

In the absence of the space-time coordinate dependence, $\beta$ becomes here global phase. At such a phase, (128) takes the form of the global gauge invariance, and the Lagrangian $L_{0}^{A}$ is invariant concerning its action.

However, at the use of the local phase $\beta(x)$, the expected structure of the Lagrangian encounters the appearance of an additional term

$$
L_{0}^{A^{\prime}}=i \bar{\psi}_{l^{A}}^{\prime} \gamma_{5} \gamma_{\mu} \partial_{\mu} \psi_{l^{A}}^{\prime}-m_{l^{A}} \bar{\psi}_{l^{A}}^{\prime} \gamma_{5} \psi_{l^{A}}^{\prime}=L_{0}+\bar{\psi}_{l^{A}} \gamma_{\mu} \partial_{\mu} \beta \psi_{l^{A}}
$$

violating its gauge invariance.

To restore this symmetry, we introduce a new axial-vector field $A_{\mu}^{A}$ which at the fulfilment of (128) has the following gauge transformation:

$$
A_{\mu}^{A^{\prime}}=A_{\mu}^{A}+\frac{1}{e_{l^{A}}} \gamma_{5} \partial_{\mu} \beta
$$

The introduced field due to his C-noninvariant nature has no interaction with a vector lepton but possesses with a truly neutral lepton a kind of axial-vector interaction. It may be expressed by the Lagrangian

$$
L_{i n t}^{A}=e_{l^{A}} j_{e m}^{A_{l}} A_{\mu}^{A}=e_{l^{A}} \bar{\psi}_{l^{A}} \gamma_{5} \gamma_{\mu} \psi_{l^{A}} A_{\mu}^{A} .
$$

Making the explicit gauge transformations, we found

$$
L_{i n t}^{A^{\prime}}=e_{l^{A}} \bar{\psi}_{l^{A}}^{\prime} \gamma_{5} \gamma_{\mu} \psi_{l^{A}}^{\prime} A_{\mu}^{A^{\prime}}=L_{i n t}^{A}-\bar{\psi}_{l^{A}} \gamma_{\mu} \partial_{\mu} \beta \psi_{l^{A}}
$$

This together with (127) convinces us here that

$$
L^{A}=\bar{\psi}_{l^{A}} \gamma_{5}\left(i \gamma_{\mu} \partial_{\mu}-m_{l^{A}}\right) \psi_{l^{A}}+e_{l^{A}} \bar{\psi}_{l^{A}} \gamma_{5} \gamma_{\mu} \psi_{l^{A}} A_{\mu}^{A}
$$

The presence of mass of field $A_{\mu}^{A}$ in the Lagrangian would imply its noninvariance concerning the chosen gauge transformation.

Thus, we have established only the part of the united Dirac interaction of C-noninvariant character in which the introduced massless field $A_{\mu}^{A}$ is equalized with an axial-vector Coulomb field. Therefore, it is not surprising that the full Lagrangian $L^{A}$ invariant concerning the local gauge transformations has the following structure:

$$
L^{A}=\bar{\psi}_{l^{A}} \gamma_{5}\left(i \gamma_{\mu} \partial_{\mu}-m_{l^{A}}\right) \psi_{l^{A}}-\frac{1}{4} F_{\mu \lambda}^{A} F_{A}^{\mu \lambda}+e_{l^{A}} \bar{\psi}_{l^{A}} \gamma_{5} \gamma_{\mu} \psi_{l^{A}} A_{\mu}^{A}
$$

The first pair of terms here corresponds to the free moving of a C-odd lepton of mass $m_{l^{A}}$, the term with a gauge-invariant tensor

$$
F_{\mu \lambda}^{A}=\partial_{\mu} A_{\lambda}^{A}-\partial_{\lambda} A_{\mu}^{A}
$$

characterizes the free axial-vector Coulomb field, and the latter term describes the minimal interaction with this field of emission of an axial-vector current

$$
j_{\mu}^{\gamma^{A}}=\bar{\psi}_{l^{A}} \gamma_{5} \gamma_{\mu} \psi_{l^{A}} .
$$


Comparing (136) with the anapole part of current (21) at $g_{1 l}=1$, it is easy to observe the correspondence which says about that an axial-vector photon appears as the field $A_{\mu}^{A}$ necessary for the creation of a theory of truly neutral fermions invariant concerning the local axial-vector gauge transformations.

These transformations are the abelian ones. In other words, each of them remains as the transformation of an abelian group $U_{A}(1)$ if $\beta(x)$ in matrix $U_{A}$ is not connected with any so far unobserved properties of matter fields violating its unitarity.

Returning to (133), we remark that the Dirac equation for a truly neutral lepton in the new field $A_{\mu}^{A}$ has the form

$$
i \gamma_{\mu} \partial_{\mu} \psi_{l^{A}}=m_{l^{A}} \psi_{l^{A}}-e_{l^{A}} \gamma_{\mu} A_{\mu}^{A} \psi_{l^{A}} .
$$

From its point of view, the divergence of an axial-vector current is equal to

$$
\partial_{\mu} j_{\mu}^{\gamma^{A}}=2 i m_{l^{A}} \bar{\psi}_{l^{A}} \gamma_{5} \psi_{l^{A}}
$$

and becomes continuity equation only in the case of the lepton mass being wholly absent.

At first sight in conformity with ideas of axial anomaly, this would have no place, since an ultimate expression [35-37] for the divergence of an axial-vector current $j_{\mu 5}$ in the conservation limit of a vector current $j_{\mu}$ is reduced to the following:

$$
\partial_{\mu} j_{\mu 5}=2 i m \bar{\psi} \gamma_{5} \psi+\frac{e^{2}}{16 \pi^{2}} \epsilon_{\mu \lambda \rho \sigma} F_{\mu \lambda} F_{\rho \sigma}
$$

where $e$ is the electron charge, $\epsilon_{0123}=1$.

On the other hand, the same lepton may not be simultaneously both a vector C-even fermion and an axial-vector C-odd one, as follows from considerations of symmetry. Therefore, we cannot exclude an axial-vector photon as the field giving in principle the possibility for the establishment in nature of continuity of an axial-vector current regardless of whether or not an unbroken vector current conservation law exists.

It is interesting, however, that the same photon of an axial-vector nature may not serve as the source of the two types of axial fields of a different character. At the same time, the existence itself of an electromagnetic field is explained by the fundamental symmetry between the electricity and the magnetism stating that to any type of the photon with the electric mass [38] and charge [39] corresponds a kind of monophoton [40] with the magnetic mass and charge. If such pairs are of axial-vector types, they constitute the naturally united axialvector electromagnetic field. But a notion about axial-vector photons of a magnetic nature was introduced [41-44] over forty years ago.

Another important prediction of a classification of elementary particles with respect to Coperation is the difference between the photons of a vector and an axial-vector nature. From its point of view, the vector photons due to their C-invariant characters have no interaction with truly neutral leptons but possess with leptons of C-even nature a kind of vector interaction.

However, in spite of this, we can accept, in the framework of the standard electroweak theory, the vector photon $\left(\gamma^{V}\right)$ as a gauge field if we make in (127)-(137) the following replacements:

$$
A \rightarrow V, \quad \gamma_{5} \rightarrow 1, \quad \beta(x) \rightarrow \alpha(x), \quad A_{\mu}^{A} \rightarrow A_{\mu}^{V}
$$

Of them $\alpha(x)$ denotes the local phase in the transformation

$$
\psi_{l^{V}}^{\prime}(x)=U_{V} \psi_{l^{V}}(x), \quad U_{V}=e^{i \alpha(x)} .
$$

Thus, only the part is obtained of the united Dirac interaction of a C-invariant nature in which a vector photon appears as the field $A_{\mu}^{V}$ necessary for the creation of a theory of Dirac 
fermions of C-even character invariant concerning the local vector gauge transformations of an unitary group $U_{V}(1)$ if $\alpha(x)$ in matrix $U_{V}$ does not possess any new properties violating its abelianity.

\section{Unity of flavour and gauge symmetry laws}

It is already clear from the above reasoning that $U_{V}(1)$ and $U_{A}(1)$ describe the internal symmetry of C-invariant and C-noninvariant fermions, and an invariance of a theory concerning the local gauge transformations leads to the appearance of the corresponding fields of emission of photons of vector and axial-vector character. Their nature has been created so that to each type of C-even or C-odd lepton corresponds a kind of flavour.

Furthermore, if these situations follow from a unified principle, flavour symmetry one must consider as a gauge symmetry [4].

To show their features, we investigate here those Lagrangians which may symbolically be written as

$$
\begin{aligned}
L_{0} & =L_{0}^{V}+L_{0}^{A}, \\
L_{i n t} & =L_{i n t}^{V}+L_{i n t}^{A} .
\end{aligned}
$$

At the same time, it is clear that (127) and (131) at the use of (140) define $L_{0}^{V}, L_{\text {int }}^{V}$ and require the elucidation of the ideas of each of flavour and gauge symmetry laws.

From the point of view of a classification of elementary particles with respect to C-operation, any of (142) and (143) assumed that $U(1) \rightarrow U_{V}(1)$ or $U_{A}(1)$. In other words, among the fermions and photons there are vector and axial-vector particles. This in turn implies that

$$
\begin{aligned}
& U_{A} \psi_{l^{V}}(x)=0, \quad U_{A} \psi_{l^{A}}(x) \neq 0, \\
& U_{V} \psi_{l^{A}}(x)=0, \quad U_{V} \psi_{l^{V}}(x) \neq 0 .
\end{aligned}
$$

Under such circumstances, a full Lagrangian is invariant concerning the local gauge transformations:

$$
L_{0}^{\prime}+L_{\text {int }}^{\prime}=L_{0}+L_{\text {int }}
$$

If we suppose here that $\psi_{l_{L, R}}=l_{L, R}$ and $\bar{\psi}_{l_{R, L}}=\bar{l}_{R, L}$, we would present the individual difermions (14) and (20) as follow:

$$
\left(\psi_{l_{L, R}^{V}}, \bar{\psi}_{l_{R, L}^{V}}\right), \quad\left(\psi_{l_{L, R}^{A}}, \bar{\psi}_{l_{R, L}^{A}}\right),
$$

in which appears a fundamental part of flavour and gauge symmetries.

In the framework of the standard electroweak theory, the same lepton must possess with vector and axial-vector fields of emission of the same types of photons simultaneously each of C-invariant and C-noninvariant types of interactions. They are of course described by the same Lagrangian that unites their in a unified whole. Therefore, from its point of view, it should be expected that $U(1) \rightarrow U_{V}(1) \otimes U_{A}(1)$. This standard unification leads us once more to an implication that

$$
\begin{array}{ll}
U_{A} \psi_{l^{V}}(x) \neq 0, & U_{A} \psi_{l^{A}}(x) \neq 0, \\
U_{V} \psi_{l^{A}}(x) \neq 0, & U_{V} \psi_{l^{V}}(x) \neq 0 .
\end{array}
$$

At these situations, (142) and (143) replace (146) by

$$
L_{0}^{\prime}+L_{\text {int }}^{\prime}=L_{0}+L_{\text {int }}+e_{l^{V}} j_{\mu} A_{\mu}^{A}+e_{l^{A}} j_{\mu 5} A_{\mu}^{V},
$$


where it has been accepted that

$$
\begin{gathered}
e_{l^{A}}=e_{l^{V}}, \quad A_{\mu}^{A} \neq A_{\mu}^{V}, \\
j_{\mu}=\bar{\psi}_{l^{V}} \gamma_{\mu} \psi_{l^{V}}, \quad j_{\mu 5}=\bar{\psi}_{l^{A}} \gamma_{5} \gamma_{\mu} \psi_{l^{A} . .}
\end{gathered}
$$

Each term with the current and the field of a different nature violates, as has been mentioned above, a gauge invariance. Their presence can explain, in the case of interaction [20] between the lepton and the field of emission, the appearance $[12,13]$ of mixed difermions (47), (48) and the following connections:

$$
\begin{array}{ll}
\left(\psi_{l_{L}^{V}}, \bar{\psi}_{l_{R}^{A}}\right), & \left(\psi_{l_{R}^{V}}, \bar{\psi}_{l_{L}^{A}}\right), \\
\left(\psi_{l_{L}^{A}}, \bar{\psi}_{l_{R}^{V}}\right), & \left(\psi_{l_{R}^{A}}, \bar{\psi}_{l_{L}^{V}}\right),
\end{array}
$$

which take place at the absence of mirror symmetry expressing the idea of flavour symmetry.

This gives the right to interpret the flavour symmetry as a gauge invariance [4]. At the same time, gauge symmetry itself must be accepted as a mirror symmetry [45].

\section{Conclusion}

Between the spaces $U_{V}(1)$ and $U_{A}(1)$ there exist fundamental differences, owing to which all elementary particles are united in families of a different C-parity. Thereby, they relate the flavour and gauge symmetries as a consequence of force unification forming the two left (right)handed leptons in individual dileptons of the same vector or axial-vector character. Such a correspondence principle can explain the absence in nature of mixed diphotons

$$
\begin{array}{cc}
\left(\gamma_{L}^{V}, \bar{\gamma}_{R}^{A}\right), & \left(\gamma_{R}^{V}, \bar{\gamma}_{L}^{A}\right), \\
\left(\gamma_{L}^{A}, \bar{\gamma}_{R}^{V}\right), & \left(\gamma_{R}^{A}, \bar{\gamma}_{L}^{V}\right)
\end{array}
$$

and the availability of a hard connection between the two left (right)-handed photons in individual diphotons

$$
\begin{array}{cc}
\left(\gamma_{L}^{V}, \bar{\gamma}_{R}^{V}\right), & \left(\gamma_{R}^{V}, \bar{\gamma}_{L}^{V}\right), \\
\left(\gamma_{L}^{A}, \bar{\gamma}_{R}^{A}\right), & \left(\gamma_{R}^{A}, \bar{\gamma}_{L}^{A}\right) .
\end{array}
$$

From this point of view, any of leptonic strings testifies in favor of the existence of a kind of bosonic string that unites the two left (right)-handed particles from the corresponding types of gauge bosons.

Therefore, the diphotons (157) and (158) together with (147) lead us to (89)-(91) once more, confirming that the nature itself does not exclude the existence of both vector and axial-vector types of fermions.

To understand their unidenticality at a more concrete experimental level, one must refer to the inequality of the absolute values of the finding bounds [33] on $\mu_{l^{V}}=f_{2 l^{V}}(0)$ and $d_{l^{A}}=$ $g_{2 l^{A}}(0)$, namely, on the sizes of the C-invariant magnetic and C-noninvariant electric dipole moments of leptons and other types of fermions, because they can appear, respectively, in the interaction vector and axial-vector structure dependence of particles and fields.

At the same time, it is clear that each type of gauge boson of a naturally united interaction constitutes a kind of current. This implies that not only the photon, but also the other boson leptonic currents include both vector C-even and axial-vector C-odd components.

In these circumstances, it seems possible to separate all gauge bosons into the two classes. The first of them consists of C-invariant vector bosons. They are of course the mediate bosons 
of vector types of interactions. We include in this class the vector photons $\gamma^{V}$ and weak $W^{ \pm}$bosons. A new example of the first group may be weak vector $Z^{ \pm}$-bosons. To the second class apply the axial-vector C-noninvariant bosons. They come forward in the system as the mediate bosons of axial-vector types of interactions. A beautiful example is axial-vector photons $\gamma^{A}$ and weak $Z^{0}\left(W^{0}\right)$-bosons. If we recognize such a behavior of the mediate bosons, accepting its ideas about that a classification of particle fundamental interactions and fields with respect to Coperation is fully compatible with gauge invariance, we would change our earlier presentations about the unified field theory of elementary particles.

Finally, insofar as the neutrino oscillation is concerned, an old picture of its building encounters the condition of a unity of flavour and gauge symmetry laws and requires the explanation both from the point of view of a classification of elementary particles with respect to C-operation and from the point of view of neutrino strings. To solve this question, it is desirable to recall at first the lepton and true flavours, the conservation of which unites the two left (right)-handed neutrinos in flavourless difermions of C-even or C-odd character. They have no definite mass. Therefore, in conformity with a gauge invariance principle, we conclude that the neutrino oscillations are carried out in the systems of the same vector or axial-vector types of neutrinos without flavour symmetry violation by the schemes

$$
\begin{gathered}
\left(\nu_{e L, R}, \bar{\nu}_{e R, L}\right) \rightarrow\left(\nu_{\mu R, L}, \bar{\nu}_{\mu L, R}\right), \\
\left(\nu_{\mu L, R}, \bar{\nu}_{\mu R, L}\right) \rightarrow\left(\nu_{\tau R, L}, \bar{\nu}_{\tau L, R}\right), \\
\left(\nu_{e L, R}, \bar{\nu}_{e R, L}\right) \rightarrow\left(\nu_{\tau R, L}, \bar{\nu}_{\tau L, R}\right) .
\end{gathered}
$$

In the presence of a purely Coulomb part of current, they correspond to the interconversions of photons of the different components:

$$
\left(\gamma_{L, R}, \bar{\gamma}_{R, L}\right) \rightarrow\left(\gamma_{R, L}, \bar{\gamma}_{L, R}\right)
$$

From such a point of view, the transitions (159)-(161) become possible until an external action separates the photon strings (157) and (158) by a part in their structural particle type dependence. Therefore, to reanalyse the experiments about mixing angles at the new level, one must elucidate the ideas of each of those oscillations which originate, for example, in the systems of the quarks and other types of hadrons.

But at a given stage we can only add that regardless of the oscillating particle types, their mixing has the unified nature. Thereby, it reflects the unity of micro-world symmetry laws.

\section{References}

1. B. Pontecorvo, B.: JETP 33, 549 (1957)

2. Fukuda, Y. et. al.: Phys. Rev. Lett. 81, 1562 (1998)

3. Wang, W.: AIP Conf. Proc. 1222, 494 (2010). arXiv:0910.4605 [hep-ex]

4. Sharafiddinov, R.S.: Bull. Am. Phys. Soc. 59(5), L1.00036 (2014)

5. Rosenbluth, M.N.: Phys. Rev. 79, 615 (1950)

6. Lee, T.D., Yang, C.N.: Phys. Rev. 104, 254 (1956)

7. Zel'dovich, Ya.B.: JETP 33, 1531 (1957) 
8. Zel'dovich, Ya.B., Perelomov, A.M.: JETP 39, 1115 (1960)

9. Landau, L.D.: JETP 32, 405 (1957)

10. Landau, L.D.: Nucl. Phys. 3, 127 (1957)

11. Sharafiddinov, R.S.: Bull. Am. Phys. Soc. 60(4), T1.00033 (2015). Spacetime Subst. 3, 86 (2002). arXiv:physics/0305009

12. Sharafiddinov, R.S.: In: Proceedings of the April Meeting of the American Physical Society, Jacksonville, Florida, April 14-17, 2007. Abstract, K11.00008

13. Sharafiddinov, R.S.: J. Phys. Nat. Sci. 4, 1 (2013). arXiv:physics/0702233

14. Sharafiddinov, R.S.: In: Proceedings of the 75th Annual Meeting of the Southeastern Section of the American Physical Society, Raleigh, North Carolina, October 29 - November 1, 2008. Abstract, NB.00009

15. Sharafiddinov, R.S.: Eur. Phys. J. Plus 126, 40 (2011). arXiv:0802.3736 [physics.gen-ph]

16. Sharafiddinov, R.S.: In: Proceedings of the April Meeting of the American Physical Society, Dallax, Texas, April 22-25, 2006. Abstract, D1.00076

17. Sharafiddinov, R.S.: Fizika B 16, 1 (2007). arXiv:hep-ph/0512346

18. Sharafiddinov,R.S.: Spacetime Subst. 3, 134 (2002). arXiv:physics/0305015

19. Yuldashev, B.S., Sharafiddinov, R.S.: Spacetime Subst. 5, 137 (2004). arXiv:hep-ph/0510080

20. Begzhanov, R.B., Sharafiddinov, R.S.: Mod. Phys. Lett. A 15, 557 (2000)

21. Greenberg, O.W.: Phys. Rev. Lett. 89, 231602 (2002). arXiv:hep-ph/0201258

22. Colladay, D., Kostelecky, A.: Phys. Rev. D 58, 116002 (1998). arXiv:hep-ph/9809521

23. Glashow, S.L.: Nucl. Phys. 22, 579 (1961)

24. Salam, A., Ward, J.C.: Phys. Lett. 13, 168 (1964)

25. Weinberg, S.: Phys. Rev. Lett. 19, 1264 (1967)

26. Chaichian, M., Fujikawa, K., Tureanu, A.: arXiv:1103.0168 [hep-th]

27. Sharafiddinov, R.S.: Phys. Essays 19, 58 (2006). arXiv:hep-ph/0407262

28. Majorana, E.: Nuovo Cimento 14, 171 (1937)

29. Sharafiddinov, R.S.: Bull. Am. Phys. Soc. 59(5), T1.00004 (2014)

30. Zel'dovich, Ya.B.: Dokl. Akad. Nauk SSSR 91, 1317 (1953)

31. Konopinsky, E.J., Mahmoud, H.: Phys. Rev. 92, 1045 (1953)

32. Sharafiddinov, R.S.: In: Proceedings of the 2rd Eurasian Conference on Nuclear Science and Its Application, Almaty, September 16-19, 2002 (Almaty, Kazakhstan, 2002), Abstracts, p. 146.

33. Yao, W.-M. et al.: Particle Data Group. J. Phys. G 33, 1 (2006) 
34. Zel'dovich, Ya.B.: Dokl. Akad. Nauk SSSR 86, 505 (1952)

35. Adler, S.L.: Phys. Rev. 177, 2426 (1969)

36. Adler, S.L., Bardeen, W.A.: Phys. Rev. 182, 1517 (1969)

37. Bell, J., Jackiw, R.: Nuovo Cimento 51, 47 (1969)

38. Prokopec, T., Tornkvist, O., Woodard, R.: Phys. Rev. Lett. 89, 101301 (2002). arXiv:astro-ph/0205331

39. Altschul, B.: Phys. Rev. Lett. 98, 261801 (2007). arXiv:hep-ph/0703126

40. Sharafiddinov, R.S.: Bull. Am. Phys. Soc. 59(5), T1.00005 (2014). Spacetime Subst. 3, 132 (2002). arXiv:physics/0305014

41. Salam, A.: Phys. Lett. 22, 683 (1966)

42. Taylor, J.D.: Phys. Rev. Lett. 18, 713 (1967)

43. Markov, M.A.: Zh. Eksp. Teor. Fiz. 3, 98 (1966)

44. Slad', L.M.: Yad. Fiz. 27, 1417 (1978)

45. Giveon, A., Witten, E.: Phys. Lett. B 332, 44 (1994). arXiv:hep-th/9404184 